\title{
Universal mechanism for air entrainment during liquid impact
}

\author{
Maurice H.W. Hendrix ${ }^{1,2}$, Wilco Bouwhuis ${ }^{1}$, Devaraj van der Meer $^{1}$, \\ Detlef Lohse $^{1}$, and Jacco H. Snoeijer ${ }^{1,3}$ \\ ${ }^{1}$ Physics of Fluids Group, Faculty of Science and Technology, University of Twente, 7500 AE \\ Enschede, The Netherlands, \\ ${ }^{2}$ Laboratory for Aero and Hydrodynamics, Delft University of Technology, Leeghwaterstraat \\ 21, NL-2628 CA Delft, The Netherlands, \\ ${ }^{3}$ Mesoscopic Transport Phenomena, Eindhoven University of Technology, Den Dolech 2, 5612 \\ AZ Eindhoven, The Netherlands
}

(Received 11 February 2015)

When a mm-sized liquid drop approaches a deep liquid pool, both the interface of the drop and the pool deform before the drop touches the pool. The build up of air pressure prior to coalescence is responsible for this deformation. Due to this deformation, air can be entrained at the bottom of the drop during the impact. We quantify the amount of entrained air numerically, using the Boundary Integral Method (BIM) for potential flow for the drop and the pool, coupled to viscous lubrication theory for the air film that has to be squeezed out during impact. We compare our results to various experimental data and find excellent agreement for the amount of air that is entrapped during impact onto a pool. Next, the impact of a rigid sphere onto a pool is numerically investigated and the air that is entrapped in this case also matches with available experimental data. In both cases of drop and sphere impact onto a pool the numerical air bubble volume $V_{b}$ is found to be in agreement with the theoretical scaling $V_{b} / V_{\text {drop } / \text { sphere }} \sim S t^{-4 / 3}$, where $S t$ is the Stokes number. This is the same scaling that has been found for drop impact onto a solid surface in previous research. This implies a universal mechanism for air entrainment for these different impact scenarios, which has been suggested in recent experimental work, but is now further elucidated with numerical results.

\section{Introduction}

The impact of a drop or a solid sphere onto a liquid pool can encompass various types of air entrainment. One possibility is that air is entrained at the top of the impacting object when the crater that is created during impact collapses, see for example Oguz \& Prosperetti (1990); Pumphrey \& Elmore (1990); Wang et al. (2013); Chen \& Guo (2014). Another type of air entrainment may occur at the bottom of the impacting object: the thin air film that is squeezed out at the impact zone is accompanied by a pressure increase that deforms the interface of the liquid before the impacting object touches the pool, which may result in air entrapment (Marston et al. 2011; Tran et al. 2013). The early stages of deformations can be described analytically (Bouwhuis et al. -). In case the impacting object is a drop, instead of a single entrapped bubble, also a collection of microscopic bubbles may be entrapped which can create intriguing morphologies (Thoroddsen et al. 2012). This is also referred to as Mesler entrainment (Esmailizadeh \& Mesler 1986; Pumphrey \& Elmore 1990). The same mechanism that is responsible for bubble entrapment at the bottom of an impacting object on a pool holds for of air entrapment at the bottom of an impacting drop onto a solid (van Dam \& Le Clerc 2004; Mani et al. 2010; 


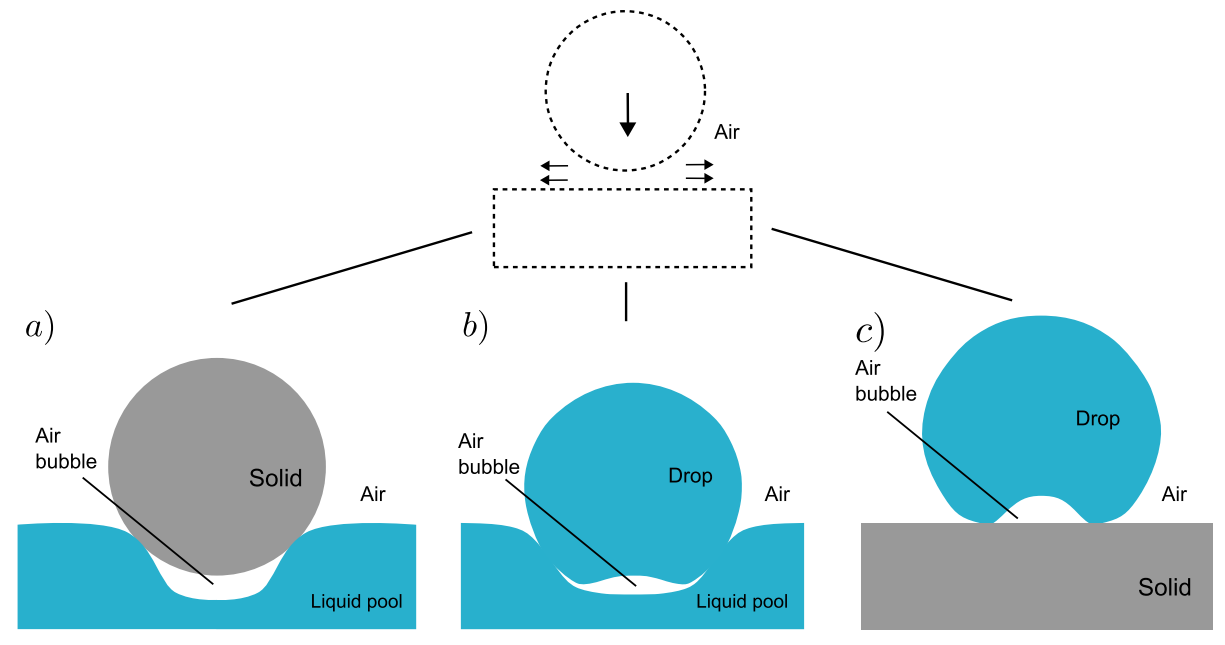

Figure 1: Air bubble entrapment for different impact scenarios. Bubbles and deformations are not drawn to scale. (a) Rigid sphere impact onto a pool. The pool deforms due to an increase in air pressure right under the sphere before it touches the pool, which results in an entrapped air bubble. (b) Drop impact onto a pool. Not only the pool, but also the drop consists of a deformable interface. As a result, the increased air pressure deforms both the pool and the drop and an air bubble is entrapped. (c) Drop impact onto a solid. Also here, a local increase in air pressure deforms the drop before it touches the solid and results in an entrapped air bubble.

Hicks \& Purvis 2010; Bouwhuis et al. 2012). In fact, the initial geometry of the problems is identical, see figure 1 in which the different impact scenarios and air entrapment have been depicted. We also refer to figure 5 of Tran et al. (2013), who first worked out this analogy.

Previously, air bubble entrapment for drop impact onto a solid surface has been quantified experimentally, theoretically, and numerically (Mandre et al. 2009; Mani et al. 2010; Hicks \& Purvis 2010, 2011; Bouwhuis et al. 2012). If the effect of surface tension can be neglected, the following scaling for the entrapped air bubble volume was found:

$$
V_{b} / V_{\text {drop }} \sim S t^{-4 / 3} .
$$

Here $V_{b} / V_{d r o p}$ is the air bubble volume normalized by the drop volume and $S t$ is the Stokes number which is defined as $S t \equiv \rho_{l} R U / \eta_{g}$, where $\rho_{l}$ is the liquid density, $R$ the droplet radius, $U$ its impact velocity, and $\eta_{g}$ is the viscosity of the surrounding gas, in this case air. The Stokes number represents the competing effect of the viscous force of the draining air film and the inertial force of the liquid which ultimately determines the air bubble volume. The same scaling was found experimentally for impact of a sphere onto a pool (Marston et al. 2011), and a drop onto a pool (Tran et al. 2013).

In this paper we try to capture the mechanism of air entrapment during impact onto a pool numerically. We will employ a boundary integral method (BIM) for potential flow describing the liquid phase coupled to viscous lubrication theory for the draining microscopic air film. The advantage of using a boundary integral method becomes evident when the interface of the impacting object comes close to the pool and one has to resolve the microscopic air layer together with the macroscopic liquid scale. This difference in length scale can be a thousandfold for the case of a millimeter sized drop impacting 
onto a pool squeezing out an air film with a typical thickness of micrometer. In fact, the difference in length scale in the final stages of impact diverges to infinity as the drop is about to coalesce with the pool. Using a boundary integral method guarantees excellent interface representation, since all variables such as liquid velocity and pressure are defined at the interface. At the same time, the computational cost is modest, since the boundary integral method allows the potential problem to be solved only at the boundaries of the liquid domain: quantities in interior points can be calculated optionally as a function of the solution at the boundary. To achieve the same accurate interface representation and solving the full Navier-Stokes equations, using for example a volume-of-fluid method (see for example Thoraval et al. (2012); Guo et al. (2014)), would require a much larger computational cost.

In section 2 we explain the theoretical framework together with the numerical method. In section 3 we will present the results of numerical simulation: we will identify details of the pressure development in the air film and deformation of the interfaces at the impact zone. The results of the numerical model will be compared with available results regarding the entrapped bubble volume from multiple experimental works and will be compared with the scaling law equation (1.1). We conclude with section 4 in which also suggestions for further research are discussed.

\section{Theory}

\subsection{Dimensional analysis and numerical method}

The Reynolds number of the liquid drops we model, which is defined as $R e_{l} \equiv \rho_{l} R U / \eta_{l}$, is assumed to be large, $R e_{l} \gg 1$. Here $\rho_{l}$ and $\eta_{l}$ are respectively the density and the dynamic viscosity of the liquid, $U$ is the impact velocity and $R$ is the radius of drop. The flow can thus be regarded irrotational, that is $\nabla \times \mathbf{u}=\mathbf{0}$. Under the additional constraint of incompressible flow inside the drop this allows the liquid dynamics to be modeled with a harmonic function $\phi$, to which the velocity field $\mathbf{u}$ is related through:

$$
\mathbf{u}=\nabla \phi
$$

The fact that the velocity potential $\phi$ obeys the Laplace equation $\nabla^{2} \phi=0$ is used to efficiently solve the potential problem, and thus the dynamics of the liquid, using the Boundary Integral Method (BIM). While the Reynolds number of the drop is large, the Reynolds number of the thin gaseous air layer $R e_{g} \equiv \rho_{g} H_{d} U / \eta_{g}$ is typically small, $R e_{g} \ll 1$. Here $\rho_{g}$ is gas density and $H_{d}$ is the air film thickness in the center of the film which is referred to as the dimple height. The length scale characterizing the air layer in the lateral extension of the air film is denoted by $L$, see figure 2a. As shown in Bouwhuis et al. (2012), $H_{d} \ll L$ which in combination with the low Reynolds number of the gas allows the film to be described with viscous lubrication theory, see for example Leal (1992). The dimensionless group reflecting the presence of air is the Stokes number $S t \equiv$ $\rho_{l} R U / \eta_{g}$ which compares the viscous force of the air layer to the inertial force in the drop. This number is relevant for describing dimple formation, since, for high enough impact velocity $U$, this process is determined by two competing forces: the force of the viscous air layer trying to deform the drop in the center and the opposing inertial force of the drop, which must be slowed down locally in order to form a dimple. Additional dimensionless numbers incorporating surface tension $\gamma$ are the Weber number $W e$ and the capillary number $\mathrm{Ca}$ based on the gas properties. Summarizing, we thus have the following dimensionless parameters: 
$R e_{l} \equiv \frac{\rho_{l} R U}{\eta_{l}} \quad R e_{g} \equiv \frac{\rho_{g} H_{d} U}{\eta_{g}} \quad S t \equiv \frac{\rho_{l} R U}{\eta_{g}} \quad W e \equiv \frac{\rho_{l} R U^{2}}{\gamma} \quad C a \equiv \frac{W e}{S t}$

The impact of a liquid drop onto a pool of the same liquid and the impact of a rigid sphere onto a liquid pool can be described with the same dimensionless numbers. As the initial geometry of the problems is identical, the difference lies in the deformability of the object, which is zero in case of the solid. The two effective control parameters that we will use here in our theoretical framework are St and We.

In figure $2 \mathrm{a}$ an illustration of the impact of a drop onto a pool, together with the used method is shown. As is clear from this figure, the coupling between the dynamics of the air layer and the dynamics of the liquid is essential since the two liquid domains feel each other through the pressure build up in the viscous air layer. The lubrication pressure $P_{g}$ acts on the liquid surface and appears in the unsteady Bernoulli equation which serves as a boundary condition in the BIM which is applied at the liquid surface, see also Bouwhuis et al. (2012). As we have two liquid domains, two separate BI equations are solved. We take the width of the pool large enough to approach the dynamics of an infinite liquid pool. In this case a width of 4.5 times the drop radius was found to be sufficient. We focus on quantifying the amount of entrapped air by integrating the enclosed air pocket up to the moment the air layer reaches a physical minimum thickness of $0.4 \mu \mathrm{m}$. At this point the volume of the enclosed air has converged and a subsequent rupture of the air film will prevent further drainage which results in an entrapped air bubble (Bouwhuis et al. 2012). As we focus on the dynamics just prior to rupture we can make use of an axisymmetric framework. We restrict ourselves to the inertial regime for which experimental results (Tran et al. 2013) are available for a direct comparison. Since the air layer continually deforms and translates during the impact, lubrication equations have been developed in a moving coordinate system which is aligned with the interface of the drop. These equations will be derived in the next section.

\subsection{Lubrication in moving and tilted coordinate system}

In this section we develop an expression for the pressure $P_{g}$ in the air film based on lubrication theory in a moving $(\|, \perp)$-coordinate system which is aligned along the drop surface, see the sketch in figure 2. The reason for doing this (rather than just using the standard $(r, z)$-coordinate system) is that especially for the drop onto pool impact, the moving $(\|, \perp)$-coordinate system is not necessarily oriented as the $(r, z)$-coordinate system and therefore only the first guarantees an accurate description of the draining air film. The drop surface is taken as a reference, and the curvilinear coordinate $\|$ is defined along the drop, starting at the axis of symmetry. At some large radial coordinate $\|_{\infty}$ we assume atmospheric pressure. The coordinate perpendicular to $\|$ is defined to be $\perp$. The gap height $h(r, t)$ is defined as the length of the perpendicular line from the drop projected onto the liquid pool. The two surfaces in the impact zone are assumed to be nearly parallel $\left(\left|\partial_{\|} h\right| \ll 1\right)$, so we can apply lubrication theory.

It can be shown (see appendix A) that the continuity equation in this new $(\|, \perp)$ coordinate system reads:

$$
\frac{u_{r}}{r}+\partial_{\|} u_{\|}+\partial_{\perp} u_{\perp}=0 .
$$

At the interface of the liquid pool $(\perp=h)$ we know that the fluid particles have to move with the interface. This is mathematically described with the kinematic boundary condition: 


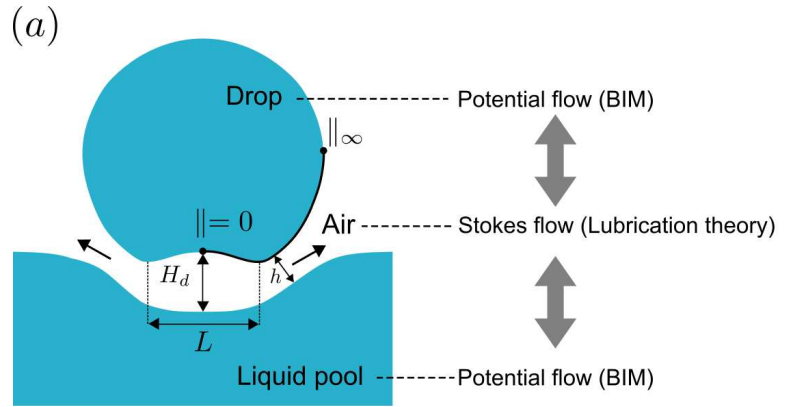

(b)

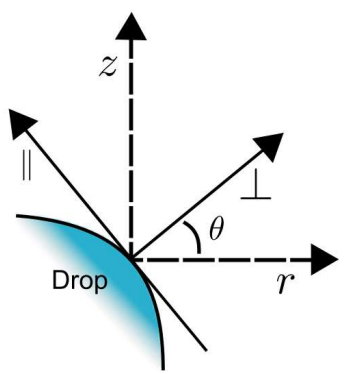

Figure 2: (a) Schematic of drop impact onto a a pool. The used methods are indicated in the figure: both the liquid domains are modelled with potential flow, while the air layer is described with Stokes flow. The gray arrows indicate that the flow of the air film is coupled to the dynamics of the liquid domains and vice versa. (b) Definition of the $(\|, \perp)$-coordinate system where $\|$ is aligned along the drop curve and $\perp$ is the unit normal with respect to the drop.

$$
\partial_{t} h+\left.\left(u_{\|} \partial_{\perp} h\right)\right|_{\perp=h}=u_{\perp \mid \perp=h}-u_{\perp \mid \perp=0} .
$$

Here $\partial_{t} h$ is the time derivative of $h$. We now integrate equation (2.3) along the gap height $h$ and obtain:

$$
\int_{0}^{h} \frac{u_{r}}{r} \mathrm{~d} \perp+\int_{0}^{h} \partial_{\|} u_{\|} \mathrm{d} \perp=-\int_{0}^{h} \partial_{\perp} u_{\perp} \mathrm{d} \perp=u_{\perp \mid \perp=0}-u_{\perp \mid \perp=h} .
$$

Using Leibniz integral rule for the second integral on the left hand side we find:

$$
\int_{0}^{h} \frac{u_{r}}{r} \mathrm{~d} \perp+\partial_{\|} \int_{0}^{h} u_{\|} \mathrm{d} \perp-\left.\left(u_{\|} \partial_{\|} h\right)\right|_{\perp=h}=u_{\perp \mid \perp=0}-u_{\perp \mid \perp=h} .
$$

We now use the kinematic boundary condition formulated in equation (2.4) for the third term on the LHS to obtain:

$$
\int_{0}^{h} \frac{u_{r}}{r} \mathrm{~d} \perp+\partial_{\|} \int_{0}^{h} u_{\|} \mathrm{d} \perp+\partial_{t} h-u_{\perp \mid \perp=h}+u_{\perp \mid \perp=0}=u_{\perp \mid \perp=0}-u_{\perp \mid \perp=h} .
$$

Canceling the terms $u_{\perp \mid \perp=h}$ and $u_{\perp \mid \perp=0}$ on both sides gives:

$$
\int_{0}^{h} \frac{u_{r}}{r} \mathrm{~d} \perp+\partial_{\|} \int_{0}^{h} u_{\|} \mathrm{d} \perp+\partial_{t} h=0 .
$$

We still have to describe $u_{r}$ within the new $(\|, \perp)$-coordinate system. Therefore we substitute $u_{r}=u_{\perp} \cos \theta-u_{\|} \sin \theta$ in the equation above to get:

$$
\int_{0}^{h} \frac{1}{r} u_{\perp} \cos \theta \mathrm{d} \perp-\int_{0}^{h} \frac{1}{r} u_{\|} \sin \theta \mathrm{d} \perp+\partial_{\|} \int_{0}^{h} u_{\|} \mathrm{d} \perp+\partial_{t} h=0 .
$$

We assume that the main flow of the air that is squeezed out from the gap is along the $\|$ coordinate, which implies that $u_{\perp}$ is relatively small, so we neglect the first term. The second term is an integral with respect to $\perp$ containing the variable $r$. This radial coordinate $r$ across $h$ is a function of $\perp: r=\perp \cos \theta+c(\|)$. Here $c(\|)$ is the value of $r$ at 
the drop surface $(\perp=0)$ for some coordinate $\|$. We thus substitute this expression for $r$ into equation (2.9) and neglect the first term to find:

$$
-\int_{0}^{h} \frac{\sin \theta}{\perp \cos \theta+c(\|)} u_{\|} \mathrm{d} \perp+\partial_{\|} \int_{0}^{h} u_{\|} \mathrm{d} \perp+\partial_{t} h=0 .
$$

\subsubsection{Flow profile within the air film}

As has been previously described the Reynolds number of the thin air film is small, $R e_{g} \ll 1$, and the geometry of the problem, $H_{d} \ll L$, allows us to use lubrication theory. In the $(\|, \perp)$-coordinate system, the Stokes equations can then be reduced to:

$$
\partial_{\|} P_{g}=\eta_{g} \partial_{\perp}^{2} u_{\|}
$$

We can integrate equation equation (2.11) twice with respect to $\perp$, employing a no slip boundary condition at the drop surface $\left(u_{\|}=U_{d}\right.$ at $\left.\perp=0\right)$ as well as at the surface of the pool $\left(u_{\|}=U_{p}\right.$ at $\left.\perp=h\right)$ :

$$
u_{\|}=\left(\left(U_{p}-U_{d}\right) \frac{\perp}{h}+U_{d}\right)+\frac{1}{2 \eta_{g}} \partial_{\|} P_{g}\left(\perp^{2}-\perp h\right) .
$$

The first term of equation (2.12) can be associated with Couette flow, caused by the movement of the interfaces. The second term can be associated with Poiseuille flow, which is driven by the radial pressure gradient, see also Klaseboer et al. (2000). Substituting this expression for $u_{\|}$in our equation for mass conservation, equation (2.10), we get:

$$
\begin{array}{r}
-\int_{0}^{h} \frac{\sin \theta}{\perp \cos \theta+c}\left[\left(\left(U_{p}-U_{d}\right) \frac{\perp}{h}+U_{d}\right)+\frac{1}{2 \eta_{g}} \partial_{\|}\left(\perp^{2}-\perp h\right)\right] \mathrm{d} \perp \\
+\partial_{\|} \int_{0}^{h}\left[\left(\left(U_{p}-U_{d}\right) \frac{\perp}{h}+U_{d}\right)+\frac{1}{2 \eta_{g}} \partial_{\|} P_{g}\left(\perp^{2}-\perp h\right)\right] \mathrm{d} \perp+\partial_{t} h=0 .
\end{array}
$$

In the first integral we deal with a prefactor $\sin \theta /(\perp \cos \theta+c)$. When taking the geometry of the problem into account we note that $\perp \cos \theta \ll c$. We can thus write $\sin \theta /(\perp$ $\cos \theta+c) \approx \sin \theta / c$. Performing the integrals of equation (2.13) under this assumption yields:

$$
-\frac{\sin \theta}{c}\left(\frac{h}{2}\left(U_{p}+U_{d}\right)-\frac{h^{3}}{12 \eta_{g}} \partial_{\|} P_{g}\right)+\partial_{\|}\left(\frac{h}{2}\left(U_{p}+U_{d}\right)-\frac{h^{3}}{12 \eta_{g}} \partial_{\|} P_{g}\right)+\partial_{t} h=0 .
$$

If we define $G(\|) \equiv\left(\frac{h}{2}\left(U_{p}+U_{d}\right)-\frac{h^{3}}{12 \eta_{g}} \partial_{\|} P_{g}\right)$ we can transform the above equation into a first order inhomogeneous linear ODE for $G(\|)$ :

$$
\dot{G}(\|)-a(\|) G(\|)=f(\|)
$$

Here $a(\|)$ and $f(\|)$ are known functions of $\|$ :

$$
\begin{aligned}
& a(\|)=\frac{\sin \theta}{c(\|)} \\
& f(\|)=-\partial_{t} h
\end{aligned}
$$


2.2.2. Solving the first order inhomogeneous $O D E$ for $G(\|)$

Equation (2.15) can be solved with help of an integrating factor $I$ defined as $I(\|) \equiv$ $\mathrm{e}^{-\int a(\|) d \|}$. Using the boundary condition $G(\|)=0$ for $\|=0$, because we have zero pressure gradient in the center of symmetry, and also zero tangential velocities, we can multiply equation $(2.15)$ with $I(\|)$ and solve for $G(\|)$ :

$$
G(\|)=\frac{1}{I(\|)}\left(\int_{0}^{\|} I(\tilde{\|}) f(\tilde{\|}) \mathrm{d} \tilde{\|}\right)
$$

with $I(\|)=\mathrm{e}^{-\int_{0}^{\|} a(\tilde{\|}) \mathrm{d} \tilde{\|}}$.

We can now substitute $G(\|) \equiv\left(\frac{h}{2}\left(U_{p}+U_{d}\right)-\frac{h^{3}}{12 \eta_{g}} \partial_{\|} P_{g}\right)$ back into equation (2.18) to obtain an equation for $\partial_{\|} P_{g}$ :

$$
\partial_{\|} P_{g}=-\frac{12 \eta_{g}}{h^{3}}\left(\frac{1}{I(\|)}\left(\int_{0}^{\|} I(\tilde{\|}) f(\tilde{\|}) \mathrm{d} \tilde{\|}\right)-\frac{h}{2}\left(U_{p}+U_{d}\right)\right)
$$

We note that we have to evaluate two numerical integrals to calculate $\partial_{\|} P_{g}$. In order to find the pressure $P_{g}(\|)$ we integrate equation (2.19) using atmospheric pressure for some large value for $\|_{\infty}$ well outside the thin air gap as a boundary value. As a check of our analysis we now orientate the $(\|, \perp)$-coordinate system in such away that $\|=r$, to recover the lubrication equation in the conventional $(r, z)$-coordinate system. In that case we have $\theta=-\pi / 2$, and we can write for $a(\|)$ :

$$
a(\|=r)=\frac{\sin \theta}{r}=-\frac{1}{r}
$$

The integrating factor $I$ now becomes:

$$
I(\|=r)=\mathrm{e}^{-\int_{0}^{r} a(\tilde{r}) d \tilde{r}}=e^{\ln r}=r
$$

Substituting equation (2.21) into equation (2.19) and using the proposition $\|=r$ and setting $U_{b}=0$ and $U_{d}=0$, we can now write equation (2.19) as:

$$
\partial_{r} P_{g}=-\frac{12 \eta_{g}}{h^{3}}\left(\frac{1}{I(r)}\left(\int_{0}^{r} I(\tilde{r}) f(\tilde{r}) \mathrm{d} \tilde{r}\right)-\frac{h}{2}\left(U_{b}+U_{d}\right)\right)=\frac{12 \eta_{g}}{h^{3}}\left(\frac{1}{r}\left(\int_{0}^{r} \tilde{r} \partial_{t} h \mathrm{~d} \tilde{r}\right)\right)
$$

We inspect that this equation (2.22) is the equation for the radial pressure gradient for viscous lubrication theory in the conventional $(r, z)$-coordinate system (Bouwhuis et al. 2013), which gives a consistency check for our analysis. This was also numerically verified.

\section{Results}

In this section simulation results will be discussed, starting with section 3.1 in which the drop impact onto a pool will be treated. The interface deformations and pressure development in the viscous air layer will be quantified. In section 3.2 we will focus on rigid sphere impact onto a pool. For both impact scenarios we will quantify the size of the air bubble that is entrapped and directly compare with various experimental results (Tran et al. 2013; Marston et al. 2011; Bouwhuis et al. 2012). In section 3.3 we will compare the dynamics of both impact scenarios and identify symmetrical behavior. 


\subsection{Drop impact onto a pool}

Figure 3a displays a typical result for drop impact onto a pool. The results are expressed in dimensional form to match the experimental conditions of the work of Tran et al. (2013), to which the numerical results in this work will be compared. In the first frame corresponding to $t=0 \mathrm{~ms}$ the initial condition of the simulation at the impact zone is shown. An initial separation of $h_{0}=50 \mu \mathrm{m}$ is used. Convergence tests regarding the initial release height have been conducted, and an initial separation of $h_{0}=50 \mu \mathrm{m}$ was found to be appropriate for the lubrication pressure to be still negligible at this distance for the parameter range which is of interest in this study. At $t=0.12 \mathrm{~ms}$ it can be seen that the pool and the drop experience the increased air pressure and thus the interfaces deform. In the lower panel of this frame, the increase in pressure is indeed visible. At $t=0.15 \mathrm{~ms}$ the drop is getting closer to the pool, and the interfaces have been further deformed. It can also be noted that the pressure maximum corresponds to a location where the separation between the drop and the pool is smallest. The location of smallest separation is now not located in the center at $r=0$ anymore. This behavior is typical for impact events involving a free surface and has been experimentally observed for e.g. drop impact onto a pool (Thoroddsen et al. 2012; Tran et al. 2013), drop impact onto a solid surface (Van der Veen et al. 2012; De Ruiter et al. 2012), sphere impact onto a pool (Marston et al. 2011) and bubble impact onto a wall in a liquid tank (Hendrix et al. 2012). In the final frame $t=0.17 \mathrm{~ms}$ we observe that the two interfaces are very close together having a minimum separation of $0.4 \mu \mathrm{m}$.

We note that the interfaces up to the final stage of impact are very well resolved, see figure $3 \mathrm{~b}$ in which the final frame at $t=0.17 \mathrm{~ms}$ is shown on various scales while keeping both axes the same length scale. In the first frame in this figure a macroscopic view of the simulation domain is shown. In the second frame the impact zone is selected and magnified. The slender geometry of the microscopic air film can be noted. In the third frame the region of closest separation is magnified. Indeed, the interfaces are very close together, the minimum separation is $0.4 \mu \mathrm{m}$. The computational nodes used for discretization of the surface are also shown in this final frame. An adaptive grid on the fluid surface allows for local refinement at the region of closest separation which results in the total number of nodes to be only of order 100, while capturing both the microscopic dynamics at the impact zone and the large scale motion of the millimeter sized drop. Note that the slanted orientation of the free surfaces in figure $3 \mathrm{~b}$ justifies the need of using a description in terms of the $(\|, \perp)$ - rather than the $(r, z)$-coordinate system.

We further note from the final frame in figure 3 a that a microscopic air film finds itself trapped between the drop and the pool. It is this entrapped air that constitutes the air bubble that is dragged into the liquid when the air film ruptures at the thinnest point and breaks the axisymmetry of the problem. In this work we do not attempt to simulate the complex rupture process of the air film itself, which is ultimately determined by surface chemistry, see for example Saylor \& Bounds (2012) who point out the importance of cleanliness of the fluid surface with respect to film rupture. Instead, we focus on the dynamics up to the rupture point, which is taken to happen at a rupture height of $0.4 \mu \mathrm{m}$. At this point, the volume of the entrained air has converged and can thus be determined, see the final frame of figure 3a. This procedure is in line with previous research (Bouwhuis et al. 2012), where experimentally the volume of the air pocket just before rupture was indeed found identical to the volume of the entrapped bubble.

\subsection{Rigid sphere impact onto a pool}

The impact of a sphere onto a pool prior to coalescence is similar to the case of a drop impacting on a pool, except that in the case of an impacting sphere the deformability of 

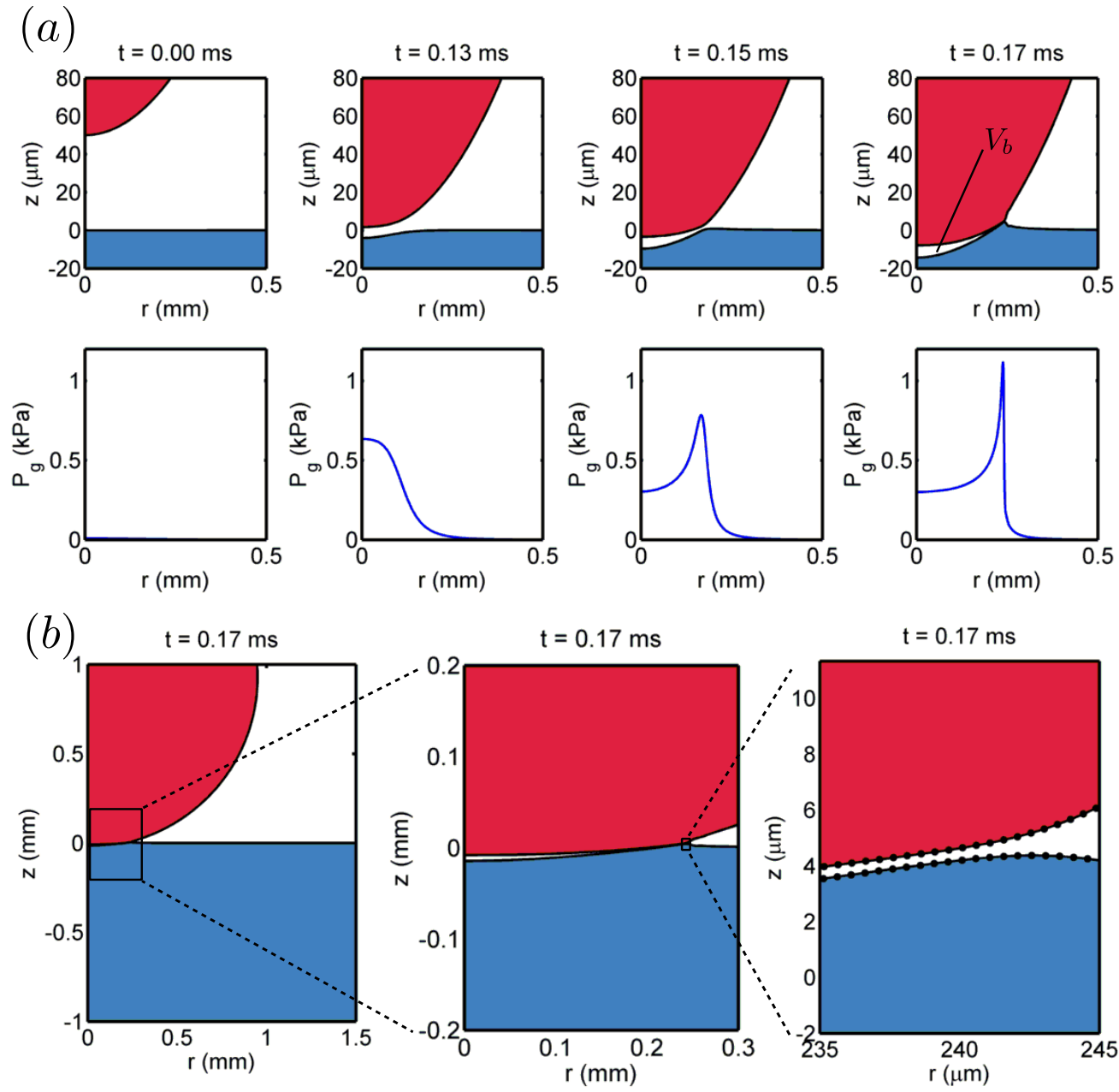

Figure 3: (a) Drop impact onto a liquid pool. Note the different length scales for the $r$-axis and $z$-axis in the shape plots. The impact speed is $U=0.42 \mathrm{~m} / \mathrm{s}$ and the drop radius is $R=0.95 \mathrm{~mm}$. The density and surface tension of the liquid are respectively $\rho=$ $916 \mathrm{~kg} / \mathrm{m}^{3}$ and $\gamma=0.020 \mathrm{~N} / \mathrm{m}$. These impact parameters correspond to $S t=2.0 \times 10^{4}$ and $W e=7.7$. The simulation starts at time $t=0 \mathrm{~ms}$ at a separation of $h_{r=0}=50 \mu \mathrm{m}$. Due to the approach of the sphere, the excess air pressure $P_{g}$ will increase and acts on both the drop and the liquid pool $(t=0.13 \mathrm{~ms})$. At the final stage $(t=0.17 \mathrm{~ms})$ the minimum separation of the interfaces reaches $0.4 \mu \mathrm{m}$ and the simulation is stopped. The bubble volume $V_{b}$ can thus be determined. (b) Part of the simulation domain with detailed snapshots of the air film at $t=0.17 \mathrm{~ms}$. In the third snapshot the actual node distribution around the smallest separation point can be inspected. This is the most refined distribution of computational nodes that is used. For the region outside the gap a coarser node distribution is sufficient. 

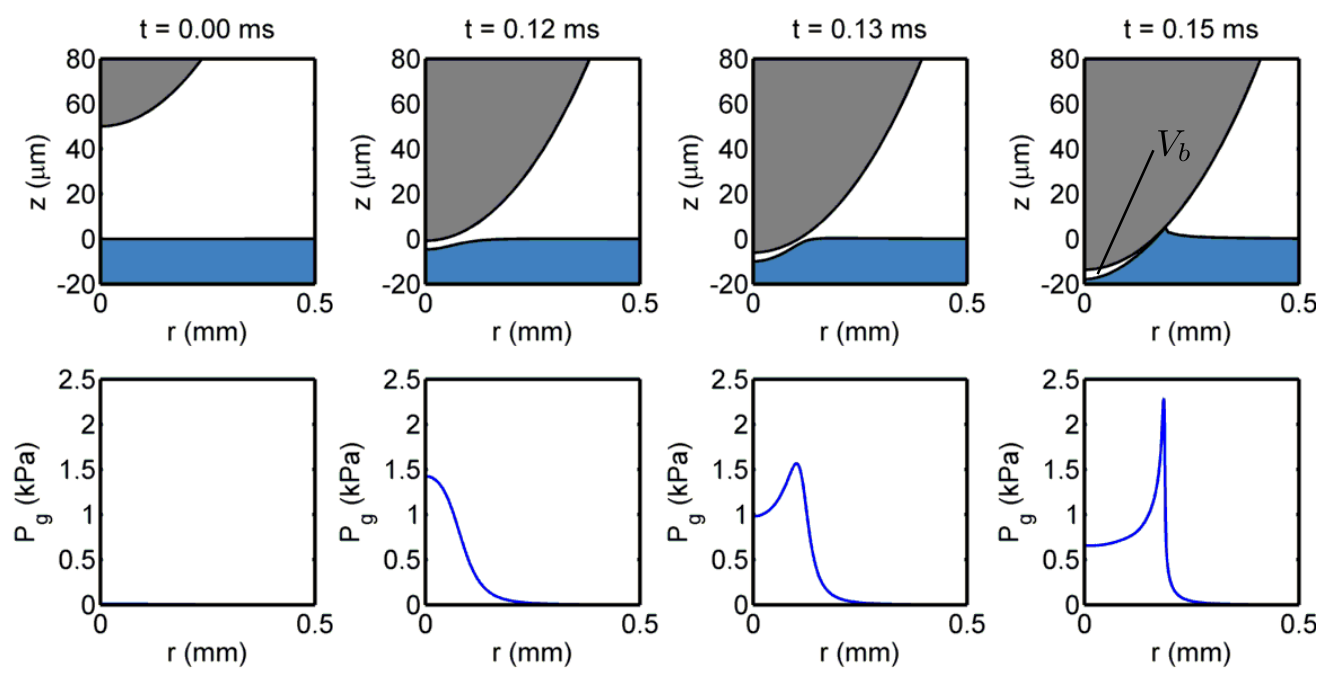

Figure 4: Rigid sphere impact onto a pool. The impact speed is $U=0.42 \mathrm{~m} / \mathrm{s}$ and the radius is $R=0.95 \mathrm{~mm}$. The density and surface tension of the fluid are respectively $\rho=$ $916 \mathrm{~kg} / \mathrm{m}^{3}$ and $\gamma=0.020 \mathrm{~N} / \mathrm{m}$. These impact parameters correspond to $S t=2.0 \times 10^{4}$ and $W e=7.7$.

the impacting object is zero. This scenario has been simulated by letting an undeformable sphere approach the pool. The same equations are solved as described in Section 2, except no BIM is needed for the impacting sphere since the interface of the sphere is fixed. The result is depicted in figure 4. Just as in the case of drop impact onto a pool, a microscopic air bubble is entrapped. As can be inspected, the air bubble has a similar shape, but its size is smaller than in case a drop impacts onto the pool, as can be inferred from a comparison to figure $3 \mathrm{a}$.

The size of the air bubble can be quantified from the numerical simulation and is compared for both drop impact and sphere impact onto a pool with various experimental results in figure 5a. We see that the numerical results of both drop and sphere impact onto a pool are in quantitative agreement with experimental work. We note that the numerical results show that the air bubble volume is indeed larger when a drop instead of a sphere impacts onto a pool for all $S t$, which is supported by experiments of Tran et al. (2013). Furthermore, we observe that numerical results are in agreement with the scaling law presented in equation (1.1), $V_{b} / V_{d r o p} \sim S t^{-4 / 3}$. As experiments have shown, in this regime, viscosity of the liquid is not important for the final bubble volume that is entrapped, see Marston et al. (2011); Tran et al. (2013), which is again confirmed by the current modeling technique which captures the essential physics which determine the air bubble volume: a potential flow calculation that does not involve liquid viscosity coupled to viscous lubrication theory for the intervening airlayer.

\subsection{Deformations of interfaces: symmetrical behavior}

We will now further investigate the fact that the bubble volume for drop impact onto a pool is larger compared to the case where we deal with only one deformable interface during impact as is the case with rigid sphere impact onto a pool. In figure 6 a closer inspection of the drop impact onto a pool is depicted. In this figure we track the relative deformation of both the pool and the drop, denoted by $\delta_{d r o p}$ and $\delta_{\text {pool }}$ respectively. 


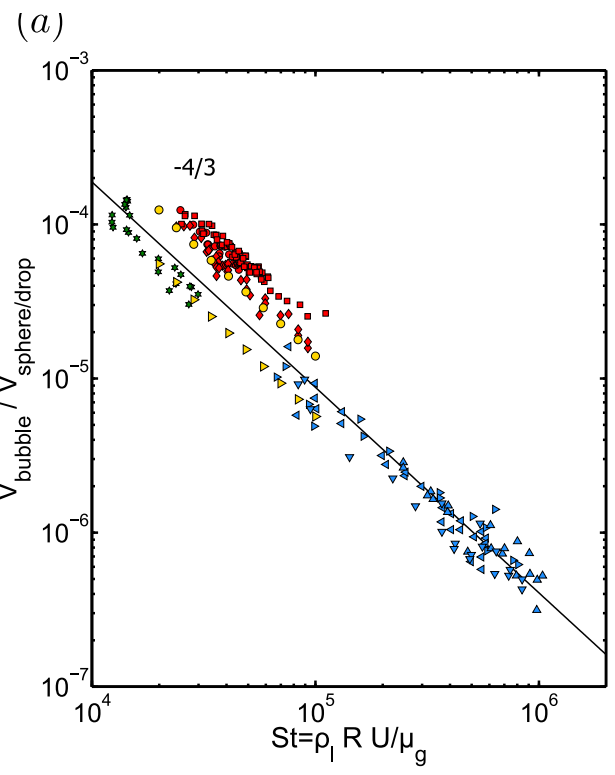

$\triangleright$ BIM, rigid sphere on pool

$\triangleleft 15 \mathrm{~mm}$ steel sphere on water (Marston et al., 2011)

$\checkmark 20 \mathrm{~mm}$ steel sphere on 10cSt oil (Marston et al., 2011)

$\Delta 20 \mathrm{~mm}$ steel sphere on $25 \mathrm{cSt}$ oil (Marston et al., 2011)

$\nabla 20 \mathrm{~mm}$ steel sphere on 1cSt SDS (Marston et al., 2011)

* Ethanol drop on solid surface (Bouwhuis et al., 2012) (b)

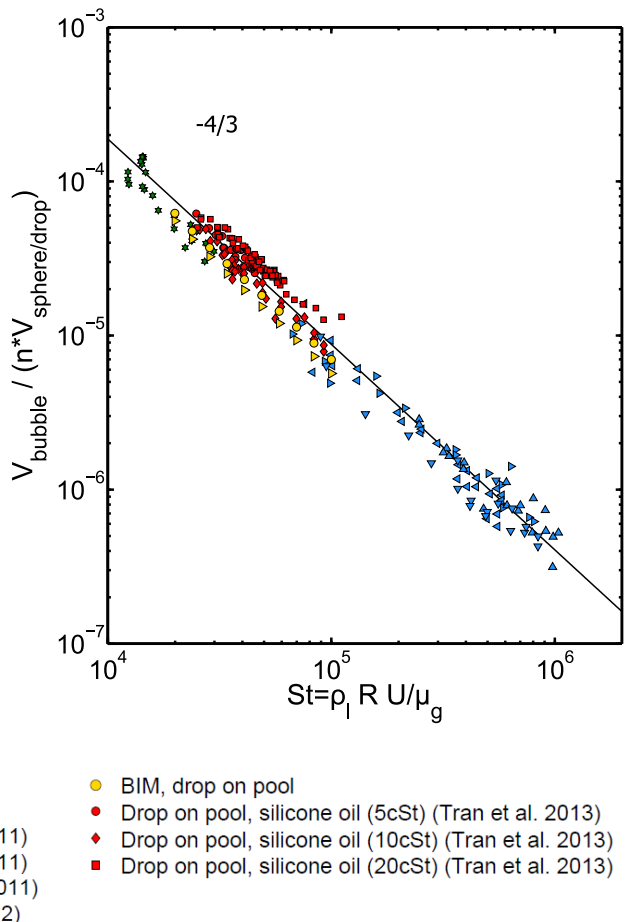

Figure 5: Figure adapted from al. Tran et al. (2013). BIM results are superimposed in yellow symbols. (a) Various experimental data for the normalized bubble volume $V_{b} / V_{\text {sphere/drop }}$ are shown. Excellent quantitative agreement was found with numerical results. (b) The data, both numerical and experimental, was found to collapse on one single curve by normalizing $V_{b}$ as $V_{b} /\left(n V_{\text {sphere }} /\right.$ drop $)$ with $n$ the number of free interfaces involved during impact. This number is 2 instead of 1 in case of drop impact onto a liquid pool.

Here $\delta_{\text {drop }}$ is defined as the deformation of the drop relative to an undeformed sphere impacting with constant speed $U$ and $\delta_{\text {pool }}$ is defined as the deformation of the pool relative to horizon $z=0$. Interestingly, we note that both interface deformations behave identically. One may expect that two deformable interfaces which react similar to an external pressure, deform in an identical way. Note, however, that the upper domain (drop) and the lower domain (pool) do not have the same unperturbed geometry, owing to the radius of curvature of the drop. Since both media respond identically to the pressure pulse, the weak curvature with respect to the width of the localized pressure has a negligible influence: on the scale of the pressure pulse, both domains are essentially flat. We therefore expect to recover a symmetric response in the upper and lower domains. To illustrate this further, we compute the kinetic energy and the velocity inside the drop and the pool using a technique described in Sun et al. (2014) to evaluate quantities close to the interface which need special attention as the singular behavior of the Green's function in the Boundary Integral equation becomes apparent for these points. Figure 7a shows the result in the frame of the pool. To highlight the symmetry, we also evaluate these quantities in a frame moving at a speed $U / 2$ in an upward direction, which results in a frame of reference in which both the drop and pool move with a speed $U / 2$ towards 

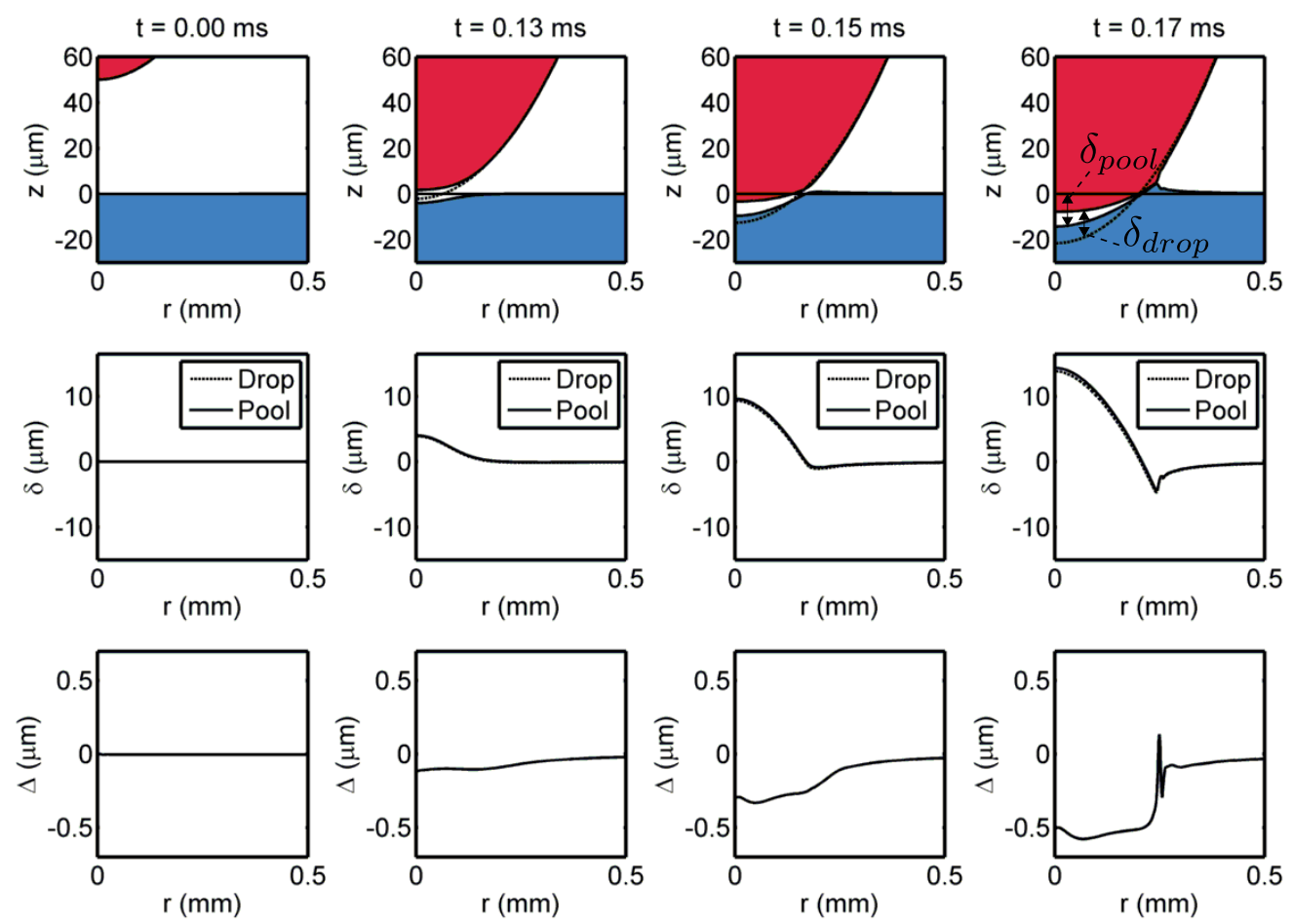

Figure 6: Drop impact onto a pool with a corresponding plot of the relative deformation $\delta$ of both the pool and the drop. In the final frame of the upper panel the definition of $\delta$ is shown. We observe excellent overlap between the relative deformations, which is emphasized in the lower panel where $\Delta=\delta_{\text {drop }}-\delta_{\text {pool }}$, the difference between the two relative deformations, is shown. The same impact conditions as for the case described in figure 3 are used: The impact speed is $U=0.42 \mathrm{~m} / \mathrm{s}$ and the radius is $R=0.95 \mathrm{~mm}$. The density and surface tension of the liquid are respectively $\rho=916 \mathrm{~kg} / \mathrm{m}^{3}$ and $\gamma=$ $0.020 \mathrm{~N} / \mathrm{m}$, which corresponds to $S t=2.0 \times 10^{4}$ and $W e=7.7$.

each other. Indeed, the velocity fields and kinetic energies are now identically distributed, see figure $7 \mathrm{~b}$.

This implies that there will be a bigger entrapped air bubble as compared to the case where only one of the interfaces is able to deform. To quantify this hypothesis we compare the bubble sizes of drop and sphere impact onto a pool and find a factor 2 difference, see figure 5b. Here half the air bubble volume of drop impact onto a pool was found to collapse onto the experimental and numerical results incorporating only one deformable interface, i.e. the sphere impact onto a pool but also drop impact onto a solid. Tran et al. (2013) took another approach to collapse the data of bubble volumes of drop impact onto a pool by correcting the corresponding impact $S t$ number by a factor 2 , which also collapses the data. In this present work it is shown that an approach based on considering the number of deformable interfaces (either 1 or 2) can also serve to obtain a unifying view on the air bubble entrapment. 


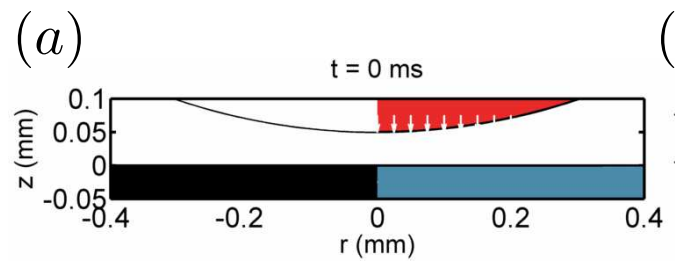

(b)
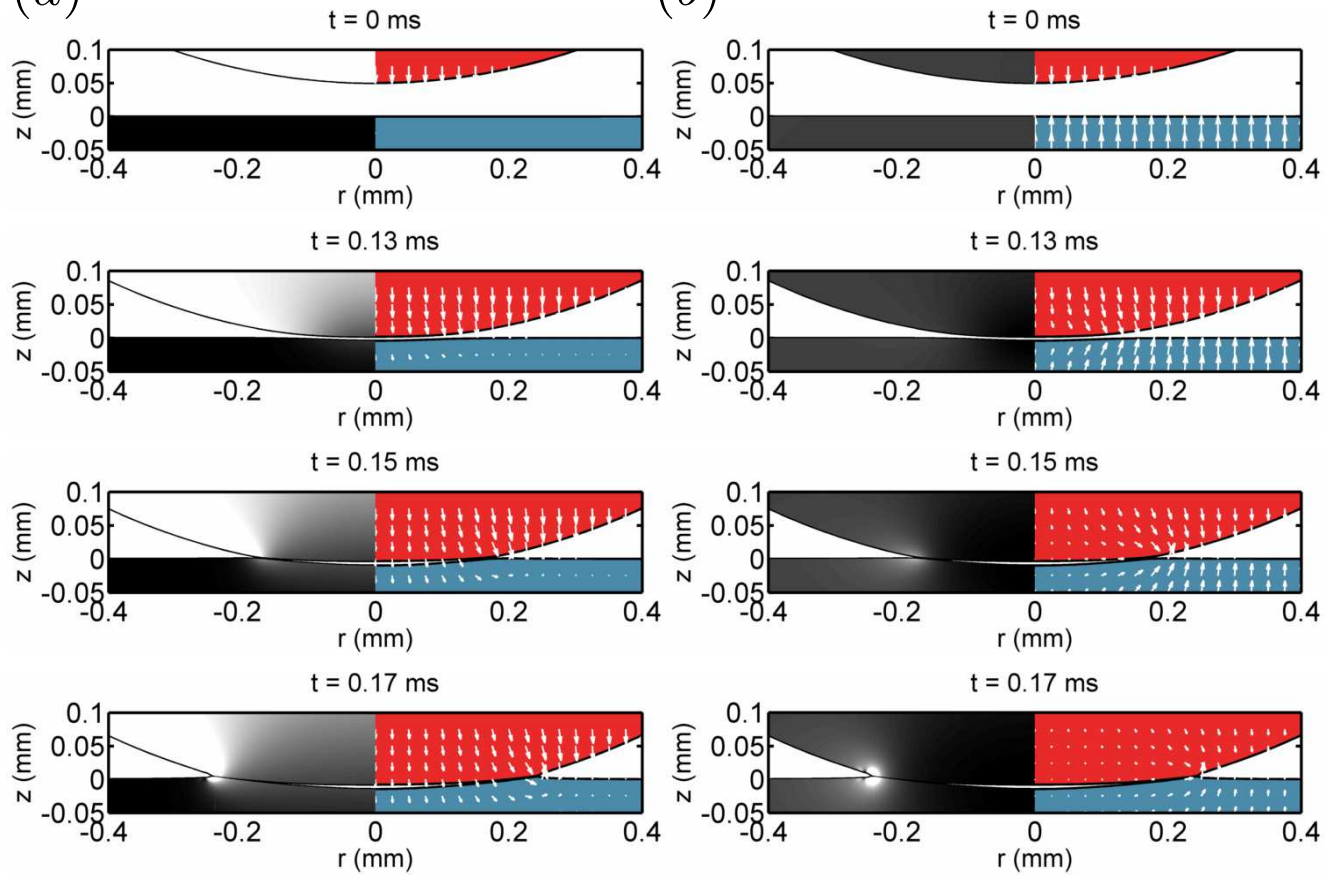

Figure 7: Kinetic energy monitoring during drop impact onto a pool, with impact parameters as described in figure 3. (a) In the left half, the kinetic energy $K$ is color coded: black is zero kinetic energy, white is maximum kinetic energy in the system which is $K=\frac{1}{2} \rho U^{2}$. (b) The kinetic energy is recalculated in a moving reference frame moving upwards at $\frac{1}{2} U$. This results in a frame of reference in which both the pool and drop move with a speed of $\frac{1}{2} U$ toward each other. Again the left half of the figure shows the kinetic energy. We observe a symmetric behavior which supports the hypothesis that the pool and drop react in a symmetric way to the local pressure increase.

\section{Conclusion}

In this work air entrapment during liquid drop and rigid sphere impact onto a deep liquid pool has been numerically investigated using a Boundary Integral Method (BIM) for potential flow for the liquid phase coupled to the viscous lubrication approximation for the subphase air which is squeezed out during impact. Excellent agreement with experimental work was found when comparing the amount of air that is entrained during impact. When considering drop impact onto a pool both liquid interfaces were found to deform identically relative to their undeformed shape. This leads to an explanation as to why bubble volumes in case of drop impact onto a pool were found to be twice the size of those that are found, both experimentally and numerically, in impacts events involving only one deformable interface, that is, rigid sphere impact onto a pool and drop impact onto a solid. In this study compressibility effects of the air have been neglected. It can be expected that at higher impact velocity compressibility of the intervening air will be important, see for example Hicks \& Purvis (2011). In addition, the current modelling technique is limited to an axisymmetric 2D framework. To account for 3D impact problems, for which experimental data starts to emerge (Van der Veen et al. 
2014), the modelling technique needs to be extended to 3D. With a 3D model also oblique collisions can be investigated.

\section{Appendix A. Continuity in curvilinear coordinates}

To derive equation 2.3 in a $(\|, \perp)$-coordinate system that moves along with the drop surface, see figure 2 , we start from the continuity equation in axisymmetric $(r, z)$ coordinates:

$$
\frac{u_{r}}{r}+\frac{\partial u_{r}}{\partial r}+\frac{\partial u_{z}}{\partial z}=0
$$

We now want to write the last two terms of the LHS of equation A 1 in terms of the $(\|, \perp)$-coordinate system, that is:

$$
\frac{u_{r}}{r}+\frac{\partial u_{r}}{\partial r}+\frac{\partial u_{z}}{\partial z}=\frac{u_{r}}{r}+\left(\frac{\partial u_{r}}{\partial \perp} \frac{\partial \perp}{\partial r}+\frac{\partial u_{r}}{\partial \|} \frac{\partial \|}{\partial r}\right)+\left(\frac{\partial u_{z}}{\partial \perp} \frac{\partial \perp}{\partial z}+\frac{\partial u_{z}}{\partial \|} \frac{\partial \|}{\partial z}\right)
$$

The two coordinate systems are related as follows (see also figure 2):

$$
\begin{array}{r}
\|=-r \sin \theta+z \cos \theta \\
\perp=r \cos \theta+z \sin \theta
\end{array}
$$

Using the relation above we can write equation A 2 as:

$$
\frac{u_{r}}{r}+\frac{\partial u_{r}}{\partial r}+\frac{\partial u_{z}}{\partial z}=\frac{u_{r}}{r}+\left(\frac{\partial u_{r}}{\partial \perp} \cos \theta-\frac{\partial u_{r}}{\partial \|} \sin \theta\right)+\left(\frac{\partial u_{z}}{\partial \perp} \sin \theta+\frac{\partial u_{z}}{\partial \|} \cos \theta\right)
$$

We now have to express $u_{r}$ and $u_{z}$ as function of $(\|, \perp)$, that is:

$$
\begin{aligned}
& u_{r}(\perp, \|)=u_{\perp}(\perp, \|) \cos \theta-u_{\|}(\perp, \|) \sin \theta \\
& u_{z}(\perp, \|)=u_{\perp}(\perp, \|) \sin \theta+u_{\|}(\perp, \|) \cos \theta
\end{aligned}
$$

Substituting the above expressions for $u_{r}$ and $u_{z}$ into equation A 5 and simplifying we find:

$$
\frac{u_{r}}{r}+\frac{\partial u_{r}}{\partial r}+\frac{\partial u_{z}}{\partial z}=\frac{u_{r}}{r}+\frac{\partial u_{\perp}}{\partial \perp}+\frac{\partial u_{\|}}{\partial \|}
$$

\section{Acknowledgments}

We gratefully acknowledge Jeremy Marston and Tuan Tran for providing their original experimental data set. This work was supported by STW and NWO through a VIDI Grant No. 11304.

\section{REFERENCES}

Bouwhuis, W., Hendrix, M. H. W., Van Der Meer, D. \& Snoeijer, J. H. - Initial surface deformations during impact on a liquid pool. http://arxiv.org/abs/1411.3576 .

Bouwhuis, W., Van Der Veen, R. C. A., Tran, T., Keij, D. L., Winkels, K. G., Peters, I. R., Van Der Meer, D., Sun, C., Snoeijer, J. H. \& Lohse, D. 2012 Maximal air bubble entrainment at liquid-drop impact. Physical Review Letters 109 (26), 264501. 
Bouwhuis, W., Winkels, K. G., Peters, I. R., Brunet, P., Van Der Meer, D. \& SnoeiJER, J. H. 2013 Oscillating and star-shaped drops levitated by an airflow. Physical Review E - Statistical, Nonlinear, and Soft Matter Physics 88 (2), 023017.

Chen, S. \& Guo, L. 2014 Viscosity effect on regular bubble entrapment during drop impact into a deep pool. Chemical Engineering Science 109, 1-16.

van Dam, D. B. \& Le Clerc, C. 2004 Experimental study of the impact of an ink-jet printed droplet on a solid substrate. Physics of Fluids 16 (9), 3403-3414.

De Ruiter, J., Оh, J. M., Van Den Ende, D. \& Mugele, F. 2012 Dynamics of collapse of air films in drop impact. Physical Review Letters 108 (7), 074505.

Esmailizadeh, L. \& Mesler, R. 1986 Bubble entrainment with drops. Journal of colloid and interface science 110 (2), 561-574.

Guo, Y., Wei, L., Liang, G. \& Shen, S. 2014 Simulation of droplet impact on liquid film with clsvof. International Communications in Heat and Mass Transfer 53, 26-33.

Hendrix, M. H. W., Manica, R., Klaseboer, E., Chan, D. Y. C. \& Ohl, C. D. 2012 Spatiotemporal evolution of thin liquid films during impact of water bubbles on glass on a micrometer to nanometer scale. Physical Review Letters 108 (24), 247803.

Hicks, P. D. \& PuRvis, R. 2010 Air cushioning and bubble entrapment in three-dimensional droplet impacts. Journal of Fluid Mechanics 649, 135-163.

Hicks, P. D. \& Purvis, R. 2011 Air cushioning in droplet impacts with liquid layers and other droplets. Physics of Fluids $\mathbf{2 3}$ (6), 062104.

Klaseboer, E., Chevaillier, J. P., Gourdon, C. \& Masbernat, O. 2000 Film drainage between colliding drops at constant approach velocity: Experiments and modeling. Journal of colloid and interface science 229 (1), 274-285.

Leal, L. GARY, ed. 1992 \{CHAPTER\} 7 - Thin Films, Lubrication, and Related Problems. Boston: Butterworth-Heinemann.

Mandre, S., Mani, M. \& Brenner, M. P. 2009 Precursors to splashing of liquid droplets on a solid surface. Physical Review Letters 102 (13), 134502.

Mani, M., Mandre, S. \& Brenner, M. P. 2010 Events before droplet splashing on a solid surface. Journal of Fluid Mechanics 647, 163-185.

Marston, J. O., Vakarelski, I. U. \& Thoroddsen, S. T. 2011 Bubble entrapment during sphere impact onto quiescent liquid surfaces. Journal of Fluid Mechanics 680, 660-670.

Oguz, Hasan N. \& Prosperetti, Andrea 1990 Bubble entrainment by the impact of drops on liquid surfaces. Journal of Fluid Mechanics 219, 143-179.

Pumphrey, Hugh C. \& Elmore, Paul A. 1990 Entrainment of bubbles by drop impacts. Journal of Fluid Mechanics 220, 539-567.

SAYlor, J. R. \& Bounds, G. D. 2012 Experimental study of the role of the weber and capillary numbers on mesler entrainment. AIChE Journal 58 (12), 3841-3851.

Sun, Q., Klaseboer, E., Khoo, B. C. \& Chan, D. Y. C. 2014 A robust and non-singular formulation of the boundary integral method for the potential problem. Engineering Analysis with Boundary Elements 43, 117-123.

Thoraval, M. ., Takehara, K., Etoh, T. G., Popinet, S., Ray, P., Josserand, C., ZaLESKi, S. \& Thoroddsen, S. T. 2012 von kármán vortex street within an impacting drop. Physical Review Letters 108 (26), 264506.

Thoroddsen, S. T., Thoraval, M. J., Takehara, K. \& Eтон, T. G. 2012 Micro-bubble morphologies following drop impacts onto a pool surface. Journal of Fluid Mechanics 708, 469-479.

Tran, T., De Maleprade, H., Sun, C. \& Lohse, D. 2013 Air entrainment during impact of droplets on liquid surfaces. Journal of Fluid Mechanics 726 (R3).

Van der Veen, R. C. A., Hendrix, M. H. W., Tran, T., Sun, C., Tsai, P. A. \& Lohse, D. 2014 How microstructures affect air film dynamics prior to drop impact. Soft Matter 10 (21), 3703-3707.

Van der Veen, R. C. A., Tran, T., Lohse, D. \& Sun, C. 2012 Direct measurements of air layer profiles under impacting droplets using high-speed color interferometry. Physical Review E - Statistical, Nonlinear, and Soft Matter Physics 85 (2), 026315.

Wang, A., Kunn, C. \& Tsai, P. 2013 Do we understand the bubble formation by a single drop impacting upon liquid surface? Physics of Fluids 25 (10), 101702. 